MATHEMATICS OF COMPUTATION

Volume 72, Number 241, Pages 435-458

S $0025-5718(02) 01422-9$

Article electronically published on May 3, 2002

\title{
CONSTRUCTING HYPERELLIPTIC CURVES OF GENUS 2 SUITABLE FOR CRYPTOGRAPHY
}

\author{
ANNEGRET WENG
}

\begin{abstract}
In this article we show how to generalize the CM-method for elliptic curves to genus two. We describe the algorithm in detail and discuss the results of our implementation.
\end{abstract}

\section{INTRODUCTION}

In 1986 Atkin [1] proposed an algorithm for primality proving using elliptic curves. An important part of his algorithm deals with the construction of elliptic curves with given group order. It is based on the theory of complex multiplication and was investigated in detail by Atkin and Morain in [2]. The complex multiplication method (short: CM-method) turned out to be a very efficient algorithm for producing elliptic curves used for cryptosystems.

Although point counting on randomly chosen elliptic curves has been improved over the years and is now sufficiently fast, the CM-method is still attractive. If the class number is not too large (say $\leq 1000$ ), the class polynomial can easily be factored. In this case the CM-method is faster than point counting. Also, the curves are constructed so as to have a suitable group order.

Koblitz [8] suggested the use of Jacobians of hyperelliptic curves for cryptography to provide a larger class of curves. For general hyperelliptic curves of small genus (say $g \leq 3$ ) the discrete logarithm problem in the Jacobian of a hyperelliptic curve is thought to be hard. Though the scalar multiplication is slower than for elliptic curves, we are working over smaller fields, which has some advantages.

To ensure the security of a cryptosystem based on the discrete logarithm problem in a finite abelian group, we have to make sure that the group order contains a large prime factor [18. More precisely, the group order should either be prime or a product of a prime and a small number. For hyperelliptic curves, finding the group order of the Jacobian seems to be a nontrivial task. Up to now there exists no point counting algorithm for randomly chosen hyperelliptic curves which reaches the group order suitable for cryptography (say $2^{160}$ ). Gaudry and Harley have recently implemented a generalisation of Schoof-Atkin-Elkies and were able to determine the order of the Jacobian of a curve of genus two defined over $\mathbb{F}_{p}$ where $p=10^{19}+51[4]$.

Received by the editor January 19, 2001 and, in revised form, March 29, 2001.

2000 Mathematics Subject Classification. Primary 11Y16, 11Y40, 94A60; Secondary 14K22, $14 \mathrm{H} 45$.

This work was supported by the NRW Forschungsverbund Datensicherheit (see www.datensicherheit.nrw.de) and the DFG (Graduiertenkolleg).

(C)2002 American Mathematical Society 
In this paper we want to discuss one possible solution to the group order problem on hyperelliptic curves over finite fields. There exists a generalisation of the elliptic curve algorithm with complex multiplication by Frey and Spallek 21] in the case of genus two. Spallek gave two examples with class number one in her thesis. Wang 23] and Weber 24] suggested replacing the computation of Gröbner bases with an efficient algorithm by Mestre [12. Based on Spallek's work, van Wamelen constructed all curves defined over $\mathbb{Q}$ having complex multiplication [22].

A complete description and implementation of the CM-method for $g=2$ does not yet exist in the literature. In this paper we combine the ideas in [21, 23, 24, 22] to get an efficient algorithm. We discuss our implementation and give examples. We were able to compute hyperelliptic curves over prime fields whose Jacobians have complex multiplication by the maximal order in a CM-field up to class number 10 . Finally we present statistics on the distribution of the group order of the Jacobian.

In Section 2 we recall some basic definitions about hyperelliptic curves over finite fields. The construction is restricted to hyperelliptic curves of genus 2 which are defined over a prime field $\mathbb{F}_{p}$ or a small extension of a prime field.

The rough idea of the algorithm goes as follows:

1. Fix a CM-field $K$ and find a suitable prime $p$ and a possible group order $n$.

2. List all principally polarized abelian varieties over $\mathbb{C}$ having complex multiplication by the maximal order $\mathcal{O}_{K}$. They are given by certain period matrices $\Omega_{i} \in \mathbb{H}_{2}$ where $\mathbb{H}_{2}=\left\{z \in \mathbb{C}^{2 \times 2}, z=z^{t}, \operatorname{Im} z>0\right\}$ is the Siegel upper half plane (see Section 3 ).

3. Compute the ten theta constants up to a certain precision (see Section 4 and the Appendix, p. 456).

4. Compute Igusa's invariants $j_{1}, j_{2}, j_{3}$ from the theta constants (see Section 5 ). Reduce them modulo $p$.

5. Compute Mestre's invariants $Q_{i j}$ and $H_{i j k}$ from $j_{1}, j_{2}, j_{3}$ (see Section 6).

6. Apply Mestre's algorithm to get the equation of the hyperelliptic curve $C$ (see Section 7).

7. Check whether the group order $\# J(C)$ is equal to $n$.

The complete algorithm is presented in Section 9 . In Section 8 we show how to get good Weil numbers for $g=2$. In Section 10 we give an analysis of the complexity of the algorithm, including a list of CM-fields suitable for our method. Section 11 lists several statistics. In Section 12 we give two examples. In Section 13 we discuss further improvements and generalisations and the limits of the CM-method.

\section{Definitions}

2.1. The Jacobian of a hyperelliptic curve. In this section we fix notations and give some basic definitions about hyperelliptic curves over finite fields and complex multiplication.

Let $C$ be a hyperelliptic curve of genus 2 defined over a finite prime field $\mathbb{F}_{p}$ $(p \neq 2)$. Then the curve can be given in the form

$$
y^{2}=f(x),
$$

where $f(x) \in \mathbb{F}_{p}[x]$ has degree six and no multiple roots in $\overline{\mathbb{F}}_{p}$.

In cryptography we consider the abelian group formed by the $\mathbb{F}_{p}$-rational points on the Jacobian of $C$. The Jacobian is defined by

$$
J_{C}=\operatorname{Div}_{0}(C) / \operatorname{Princ}(C)
$$


where $\operatorname{Div}_{0}(C)$ (resp. Princ $(C)$ ) denotes the group of degree 0 divisors (resp. principal divisors) (see [8]). We denote the group of $\mathbb{F}_{p}$-rational points on its Jacobian by $J_{C}\left(\mathbb{F}_{p}\right)$.

\subsection{The Frobenius of hyperelliptic curves of genus two over finite fields.}

The isogeny

$$
(x, y) \mapsto\left(x^{p}, y^{p}\right)
$$

on the curve $C$ induces an endomorphism $\pi$ on the Jacobian $J_{C}$. The endomorphism $\pi$ is called the Frobenius endomorphism. The characteristic polynomial of the Frobenius is a polynomial of degree 4 . If the characteristic polynomial of $\pi$ is irreducible, it defines a CM-field $K$ of degree 4 over $\mathbb{Q}$. This means $K$ is an imaginary quadratic extension over a real quadratic number field $K_{0}$.

Once we know the roots $\pi_{i}$ of the characteristic polynomial we can determine the group order by

$$
\# J(C)=\prod_{i=1}^{4}\left(1-\pi_{i}\right)
$$

We fix a CM-field $K$ and consider an algebraic number $w_{1} \in K$ such that

$$
\left[\mathbb{Q}\left(w_{1}\right): \mathbb{Q}\right]=4 \text { and } w_{1} \overline{w_{1}}=p .
$$

Its conjugates are given by $w_{i}, i=2, \ldots, 4$.

Let $\operatorname{Tor}(U)$ be the torsion group of units of $K$.

Now suppose we have a curve defined over $\mathbb{F}_{p}$ having complex multiplication by $\mathcal{O}_{K}$ (the maximal order in $K$ ). Then we have at most

$$
\begin{cases}\# \operatorname{Tor}(U) & \text { if } p \text { is inert with respect to } K_{0} / \mathbb{Q} \text { or } K \text { is Galois, } \\ 2 \# \operatorname{Tor}(U) & \text { if } p \text { splits completely in } K \text { and } K \text { is not Galois }\end{cases}
$$

possibilities for the group order $\# J\left(\mathbb{F}_{p}\right)$.

From now on we assume that $K$ does not contain a cyclotomic field. Then there are two, resp. four possibilities for the group order.

\section{Complex multiplication}

In this section we refer to the literature [19], [10], [9], [21]. We will only give the definitions that are necessary to understand the algorithm.

Every abelian variety of dimension $n$ over $\mathbb{C}$ is isomorphic to $\mathbb{C}^{n} / L$ for some lattice $L$. Further, we know that there exists a nondegenerate Riemann form on the lattice $L$ (for definitions and proofs, see [10, 21]). The Riemann form induces a polarization on $L$. If it is a principal polarization the lattice can be given by $\mathbb{Z}^{n}+\Omega \mathbb{Z}^{n}$, where $\Omega$ lies in the Siegel upper half plane $\mathbb{H}_{n}=\left\{z \in M_{n}(\mathbb{C}), z^{t}=\right.$ $z, \operatorname{Im} z$ positive definite $\}$. Every Jacobian variety has a principal polarization 14 .

Let $\operatorname{End}(A)$ be the endomorphism ring of a simple abelian variety over $\mathbb{C}$. The field $\operatorname{End}(A) \otimes \mathbb{Q}$ is either a totally real number field or an imaginary quadratic extension of a totally real number field [19].

We concentrate on the case that

$$
\operatorname{End}(A) \otimes \mathbb{Q}=K \quad \text { and } \quad[K: \mathbb{Q}]=2 n
$$

and there exists a subfield $K_{0}$ in $K$ such that $K_{0}$ is totally real and $\left[K: K_{0}\right]=2$. The field $K$ is called a CM-field. 
If $\left\{\sigma_{1}, \ldots, \sigma_{n}\right\}$ are the real embeddings of $K_{0}$, then the embeddings of $K$ into $\mathbb{C}$ are given by

$$
\left\{\hat{\sigma}_{1}, \ldots, \hat{\sigma}_{n}, \rho \hat{\sigma}_{1}, \ldots, \rho \hat{\sigma}_{n}\right\}
$$

where $\hat{\sigma}_{i}$ is an embedding with $\hat{\sigma}_{i} \mid K_{0}=\sigma_{i}$ and $\rho$ denotes the complex conjugation.

We choose a subset $\Phi=\left(\varphi_{1}, \ldots, \varphi_{n}\right)$ such that $\varphi_{i} \neq \varphi_{j}, \rho \varphi_{j}$. Then $(K, \Phi)$ is called a CM-type.

Let $\mathfrak{A}$ be an ideal in $K$ and $(K, \Phi)$ a CM-type. Then

$$
\Phi(\alpha):=\left(\varphi_{1}(\alpha), \ldots, \varphi_{n}(\alpha)\right)^{t}, \quad \alpha \in \mathfrak{A},
$$

is a lattice in $\mathbb{C}^{n}$ having complex multiplication by $\mathcal{O}_{K}$, i.e., the matrix

$$
S_{\Phi}(\gamma)=\left(\begin{array}{ccc}
\varphi_{1}(\gamma) & & \\
& \ldots & \\
& & \varphi_{n}(\gamma)
\end{array}\right), \quad \gamma \in \mathcal{O}_{K},
$$

leaves the lattice invariant (see [19]). The corresponding abelian variety is said to be an abelian variety of CM-type $(K, \Phi)$.

An abelian variety of CM-type $(K, \Phi)$ is simple iff the CM-type is primitive. There exists an easy criterion for whether a CM-type is primitive (see [19], [10, [9], [21]).

Now we adapt the theory to our situation:

Note that Jacobians of hyperelliptic curves of genus 2 are exactly the principally polarized abelian varieties of dimension 2 [13.

Let $K_{0}=\mathbb{Q}(\sqrt{d}), d \in \mathbb{N}$, be a real quadratic number field of class number one. Suppose $\alpha=a+b \sqrt{d}$ is squarefree and totally positive (i.e., $a \pm b \sqrt{d}>0$ ). Then $K=\mathbb{Q}(i \sqrt{\alpha})$ is a CM-field of degree 4 over $\mathbb{Q}$. A CM-type $(K, \Phi)$ is not primitive iff $K$ is Galois with Galois group $\mathbb{Z} / 2 \mathbb{Z} \times \mathbb{Z} / 2 \mathbb{Z}$.

Let $\epsilon$ be the fundamental unit of $K_{0}$. We consider the subgroup of units which consists of all totally positive units in $K_{0}$, and denote it by $U^{+}$. It has a subgroup $U_{N}^{+}$that consists of all units which satisfy a relative norm equation with respect to $K / K_{0}$. Note that $U^{+}=U_{N}^{+}$if the fundamental unit has negative norm.

Since $K_{0}$ has class number one, the ring of integers can be given in the form

$$
\mathcal{O}_{K_{0}}+\gamma \mathcal{O}_{K_{0}}
$$

where $\gamma \in \mathcal{O}_{K}$. Further, every ideal $\mathfrak{A}_{j}$ in $\mathcal{O}_{K}$ has a relative basis

$$
\alpha_{j} \mathcal{O}_{K_{0}}+\beta_{j} \mathcal{O}_{K_{0}}, \quad \alpha_{j}, \beta_{j} \in K
$$

The ideal $\mathfrak{A}_{j}$ is equivalent to an ideal of the form

$$
\mathcal{O}_{K_{0}}+\tau_{j} \mathcal{O}_{K_{0}}, \quad \tau_{j}=\frac{\alpha_{j}}{\beta_{j}} \in \mathcal{O}_{K},
$$

where $\operatorname{Im} \tau_{j}>0$. If $\epsilon \notin U^{+}$, we will assume that $N_{K / K_{0}}\left(\tau_{j}\right)$ is always totally positive.

We can define two continuations of the real conjugation in $K_{0}$ to $K$. We set

$$
\hat{\sigma}\left(i \sqrt{\alpha}^{+}\right)=i \sqrt{\sigma(\alpha)}^{+} \text {and } \rho \hat{\sigma}\left(i \sqrt{\alpha}^{+}\right)=-i \sqrt{\sigma(\alpha)}^{+},
$$

where $\sqrt{a}^{+}$denotes the positive square root of $a \in \mathbb{R}$.

Spallek [21] proved the following theorem: 
Theorem 3.1. Let $K$ be a $C M$-field as above and $K$ either not Galois or Galois with Galois group $\mathbb{Z} / 4 \mathbb{Z}$. Let $K_{0}=\mathbb{Z}+\mathbb{Z} w$ be the real subfield, $\sigma$ the real conjugation and $\varphi=\rho \hat{\sigma}$.

1. A complete system of simple principally polarized abelian varieties (up to isomorphism) having complex multiplication by $\mathcal{O}_{K}$ is given by $\mathcal{K}=\mathcal{K}_{1, \varphi} \cup \mathcal{K}_{1, \bar{\varphi}}$, where

$$
\begin{aligned}
& \mathcal{K}_{1, \varphi}= \begin{cases}\left\{\left(\tau_{j}, \tau_{j}^{\varphi}\right): N_{K / K_{0}}\left(\tau_{j}\right) \text { totally pos. }\right\}, & \epsilon \in U_{N}^{+}, \\
\left\{\left(\tau_{j}, \tau_{j}^{\varphi}\right),\left(\epsilon \tau_{j}, \epsilon \tau_{j}^{\varphi}\right): N_{K / K_{0}}\left(\tau_{j}\right) \text { totally pos. }\right\}, & \epsilon \in U^{+}-U_{N}^{+}, \\
\left\{\left(\tau_{j}, \tau_{j}^{\varphi}\right): N_{K / K_{0}}\left(\tau_{j}\right) \text { totally pos. }\right\}, & \text { otherwise, }\end{cases} \\
& \mathcal{K}_{1, \bar{\varphi}}= \begin{cases}\emptyset, & \text { if } K \text { is Galois }, \\
\left\{\left(\tau_{j},\left(\tau_{j}\right)^{\rho \varphi}\right): N_{K / K_{0}}\left(\tau_{j}\right) \text { not totally pos. }\right\}, & \epsilon \in U_{N}^{+}, \\
\left\{\left(\tau_{j}, \tau_{j}^{\rho \varphi}\right),\left(\epsilon \tau_{j}, \epsilon \tau_{j}^{\rho \varphi}\right): N_{K / K_{0}}\left(\tau_{j}\right) \text { totally pos. }\right\}, & \epsilon \in U^{+}-U_{N}^{+}, \\
\left.\left\{\left(\epsilon \tau_{j},\left(\epsilon \tau_{j}\right)\right)^{\rho \varphi}\right): N_{K / K_{0}}\left(\tau_{j}\right) \text { totally pos. }\right\}, & \text { otherwise. }\end{cases}
\end{aligned}
$$

2. Given a principally polarized abelian variety of type $(K,\{1, \psi\})$ of the form $\left(s_{j}, s_{j}^{\psi}\right)$, the corresponding period matrix is given by

$$
\Omega_{s_{j}, s_{j}^{\psi}}=\frac{1}{w-w^{\sigma}}\left(\begin{array}{cc}
w^{2} s_{j}-\left(w^{\psi}\right)^{2} s_{j}^{\psi} & w s_{j}-w^{\psi} s_{j}^{\psi} \\
w s_{j}-w^{\psi} s_{j}^{\psi} & s_{j}-s_{j}^{\psi}
\end{array}\right) .
$$

\section{Computing the theta constants}

Once we have a period matrix $\Omega$, we want to compute the invariants of the curves corresponding to the Jacobian represented by $\Omega$. For this we use the theta constants

$$
\theta\left[\begin{array}{l}
\delta \\
\epsilon
\end{array}\right](\Omega, 0)=\sum_{n \in \mathbb{Z}^{2}} \exp \left(\pi i\left(n+\frac{1}{2} \delta\right)^{t} \Omega\left(n+\frac{1}{2} \delta\right)+2\left(n+\frac{1}{2} \delta\right)^{t}\left(\frac{1}{2} \epsilon\right)\right),
$$

where $\delta, \epsilon \in\{0,1\}^{2}$. It can easily be shown that

$$
\theta\left[\begin{array}{l}
\delta \\
\epsilon
\end{array}\right](\Omega, 0)=0
$$

if $\delta \epsilon^{t}=1 \bmod 2$. Thus we concentrate on the 10 even theta constants

$$
\theta\left[\begin{array}{l}
\delta \\
\epsilon
\end{array}\right](\Omega, 0), \quad \delta^{t} \epsilon \equiv 0 \quad \bmod 2 .
$$

They are given by

$$
\begin{aligned}
& \left.\left.\left.\left.\left.\theta_{1}:=\theta\left[\begin{array}{l}
0 \\
0 \\
0 \\
0
\end{array}\right)\right], \theta_{2}:=\theta\left[\begin{array}{l}
0 \\
0 \\
(1 \\
0
\end{array}\right)\right], \theta_{3}:=\theta\left[\begin{array}{l}
0 \\
0 \\
0 \\
0 \\
1
\end{array}\right)\right], \theta_{4}:=\theta\left[\begin{array}{l}
0 \\
0 \\
(1 \\
1
\end{array}\right)\right], \theta_{5}:=\theta\left[\begin{array}{l}
1 \\
0 \\
0 \\
0
\end{array}\right)\right],
\end{aligned}
$$

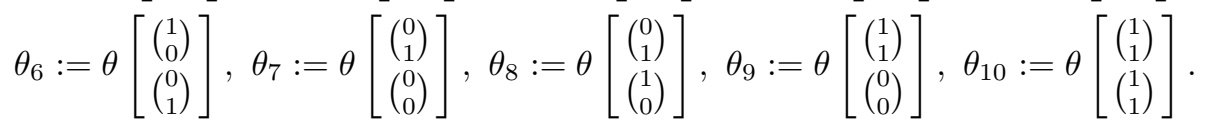

Suppose we would like to compute the theta constants up to the precision $10^{-s}$ for some integer $s$. Note that the absolute value of a summand in (1) depends only on

$$
\left|\exp \left(-\pi\left(n+\frac{1}{2} \delta\right)^{t} \operatorname{Im} \Omega\left(n+\frac{1}{2} \delta\right)\right)\right| .
$$


Thus we need an algorithm which computes all $n \in \mathbb{Z}^{2}$ such that

$$
\left(n+\frac{1}{2} \delta\right)^{t} \operatorname{Im} \Omega\left(n+\frac{1}{2} \delta\right) \leq C .
$$

Such an algorithm is given in the Appendix.

Now we have to find the value $C$ such that

$$
\left|\sum_{\substack{n \in \mathbb{Z}^{2} \\\left(n+\frac{1}{2} \delta\right)^{t} \operatorname{Im} \Omega\left(n+\frac{1}{2} \delta\right)>C}} \exp \left(\pi i\left(\left(n+\frac{1}{2} \delta\right)^{t} \Omega\left(n+\frac{1}{2} \delta\right)+2\left(n+\frac{1}{2} \delta\right)^{t}\left(\frac{1}{2} \epsilon\right)\right)\right)\right| \leq 10^{-s} .
$$

We have

$$
\begin{aligned}
& \left|\sum_{\substack{n \in \mathbb{Z}^{2} \\
\left(n+\frac{1}{2} \delta\right)^{t} \operatorname{Im} \Omega\left(n+\frac{1}{2} \delta\right)>C}} \exp \left(\pi i\left(\left(n+\frac{1}{2} \delta\right)^{t} \Omega\left(n+\frac{1}{2} \delta\right)+2\left(n+\frac{1}{2} \delta\right)^{t}\left(z+\frac{1}{2} \epsilon\right)\right)\right)\right| \\
& \leq \sum_{\substack{n \in \mathbb{Z}^{2} \\
\left(n+\frac{1}{2} \delta\right)^{t} \operatorname{Im} \Omega\left(n+\frac{1}{2} \delta\right)>C}}\left|\exp \left(-\pi\left(n+\frac{1}{2} \delta\right)^{t} \operatorname{Im} \Omega\left(n+\frac{1}{2} \delta\right)\right)\right| \\
& \leq \sum_{m=C}^{\infty} \mu(m) \exp (-\pi m),
\end{aligned}
$$

where

$$
\mu(m)=\#\left\{n \in \mathbb{Z}^{2}: m<\left(n+\frac{1}{2} \delta\right)^{t} \operatorname{Im} \Omega\left(n+\frac{1}{2} \delta\right) \leq m+1\right\} .
$$

To approximate the size of $\mu(m)$ we make use of the volume heuristics

$$
\mu(m) \approx \frac{\operatorname{Vol}\left(K_{2}(m)\right)}{(\min \Omega)^{2}},
$$

where $K_{2}(m)=\left\{z \in \mathbb{C}^{2}: m \leq\|z\| \leq m+1\right\}$ and $\min \Omega=\min _{g \in \mathbb{Z}^{2}}\left\{g^{t}(\operatorname{Im} \Omega) g\right\}$. As a rough estimate we get

$$
\operatorname{Vol}\left(K_{2}(m)\right) \leq 7 m
$$

Now we have

$$
\sum_{m=C}^{\infty} \mu(m) \exp (-\pi m) \approx \frac{7}{(\min \Omega)^{2}} \sum_{m=C}^{\infty} m x^{-m}, \quad \text { where } x=\exp (\pi) .
$$

Since the function $f(x)=x \exp (-\pi x)$ is strictly decreasing for a variable $x>1$, we have

$$
\sum_{m=C}^{\infty} m x^{-m} \leq \int_{C-1}^{\infty} m x^{-m} d m
$$


Setting $x=\exp (\pi)$ gives us the following estimate:

$$
\begin{aligned}
& \frac{7}{(\min \Omega)^{2}} \sum_{m=C}^{\infty} m x^{-m} \\
& \leq \frac{7}{(\min \Omega)^{2}} \int_{m=C-1}^{\infty} m x^{-m} d m \\
& \quad=\frac{7}{(\min \Omega)^{2}}\left((C-1) \frac{\exp (-\pi(C-1))}{\pi}+\frac{1}{\pi^{2}} \exp (-\pi(C-1))\right. \\
& <\frac{7}{(\min \Omega)^{2}}\left(\frac{C}{\pi} \exp (-\pi(C-1))\right)<\frac{2.3}{(\min \Omega)^{2}} \exp \left(-\frac{3}{4} \pi C\right)
\end{aligned}
$$

The last step uses the inequality

$$
C \exp (-\pi(C-1))<\exp \left(-\frac{3 \pi}{4} C\right) \quad \text { for } C \geq 7 .
$$

We get the following condition on $C$ :

$$
C>\left(s+0.35-2 \log _{10}(\min \Omega)\right)
$$

and

$$
C>\frac{1}{2}\left(s+0.35-2 \log _{10}(\min \Omega)\right) \quad \text { if } C \geq 75 .
$$

It is clear that a formula for $C$ will always depend on the first successive minima of $\Omega$.

\section{From theta constants to Igusa's invariants}

Given the value of the 10 even theta characteristics evaluated at the period matrix $\Omega$, we can compute the three $j$-invariants of the corresponding hyperelliptic curve.

First we define the values $h_{4}, h_{10}, h_{12}$ and $h_{16}$ coming from modular forms of weight $4,10,12$ and 16 :

$$
\begin{aligned}
h_{4}:= & \sum_{i=1}^{10} \theta_{i}^{8}, \\
h_{10}:= & \prod_{i=1}^{10} \theta_{i}^{2}, \\
h_{12}:= & \left(\theta_{1} \theta_{5} \theta_{2} \theta_{9} \theta_{6} \theta_{10}\right)^{4}+\left(\theta_{1} \theta_{2} \theta_{9} \theta_{6} \theta_{8} \theta_{3}\right)^{4}+\left(\theta_{5} \theta_{9} \theta_{6} \theta_{8} \theta_{10} \theta_{7}\right)^{4}+\left(\theta_{5} \theta_{2} \theta_{6} \theta_{8} \theta_{3} \theta_{7}\right)^{4} \\
& +\left(\theta_{1} \theta_{5} \theta_{2} \theta_{10} \theta_{3} \theta_{7}\right)^{4}+\left(\theta_{1} \theta_{9} \theta_{8} \theta_{10} \theta_{3} \theta_{7}\right)^{4}+\left(\theta_{1} \theta_{5} \theta_{2} \theta_{8} \theta_{10} \theta_{4}\right)^{4}+\left(\theta_{1} \theta_{5} \theta_{9} \theta_{8} \theta_{3} \theta_{4}\right)^{4} \\
& +\left(\theta_{5} \theta_{9} \theta_{6} \theta_{10} \theta_{3} \theta_{4}\right)^{4}+\left(\theta_{2} \theta_{6} \theta_{8} \theta_{10} \theta_{3} \theta_{4}\right)^{4}+\left(\theta_{1} \theta_{2} \theta_{9} \theta_{6} \theta_{7} \theta_{4}\right)^{4}+\left(\theta_{1} \theta_{5} \theta_{6} \theta_{8} \theta_{7} \theta_{4}\right)^{4} \\
& +\left(\theta_{2} \theta_{9} \theta_{8} \theta_{10} \theta_{7} \theta_{4}\right)^{4}+\left(\theta_{5} \theta_{2} \theta_{9} \theta_{3} \theta_{7} \theta_{4}\right)^{4}+\left(\theta_{1} \theta_{6} \theta_{10} \theta_{3} \theta_{7} \theta_{4}\right)^{4},
\end{aligned}
$$




$$
\begin{aligned}
& h_{16}:=\theta_{8}^{4}\left(\theta_{1} \theta_{5} \theta_{2} \theta_{9} \theta_{6} \theta_{8} \theta_{10}\right)^{4}+\theta_{5}^{4}\left(\theta_{1} \theta_{5} \theta_{2} \theta_{9} \theta_{6} \theta_{8} \theta_{3}\right)^{4}+\theta_{10}^{4}\left(\theta_{1} \theta_{2} \theta_{9} \theta_{6} \theta_{8} \theta_{10} \theta_{3}\right)^{4} \\
& +\theta_{3}^{4}\left(\theta_{1} \theta_{5} \theta_{2} \theta_{9} \theta_{6} \theta_{10} \theta_{3}\right)^{4}+\theta_{1}^{4}\left(\theta_{1} \theta_{5} \theta_{9} \theta_{6} \theta_{8} \theta_{10} \theta_{7}\right)^{4}+\theta_{2}^{4}\left(\theta_{5} \theta_{2} \theta_{9} \theta_{6} \theta_{8} \theta_{10} \theta_{7}\right)^{4} \\
& +\theta_{1}^{4}\left(\theta_{1} \theta_{5} \theta_{2} \theta_{6} \theta_{8} \theta_{3} \theta_{7}\right)^{4}+\theta_{9}^{4}\left(\theta_{5} \theta_{2} \theta_{9} \theta_{6} \theta_{8} \theta_{3} \theta_{7}\right)^{4}+\theta_{9}^{4}\left(\theta_{1} \theta_{5} \theta_{2} \theta_{9} \theta_{10} \theta_{3} \theta_{7}\right)^{4} \\
& +\theta_{6}^{4}\left(\theta_{1} \theta_{5} \theta_{2} \theta_{6} \theta_{10} \theta_{3} \theta_{7}\right)^{4}+\theta_{5}^{4}\left(\theta_{1} \theta_{5} \theta_{9} \theta_{8} \theta_{10} \theta_{3} \theta_{7}\right)^{4}+\theta_{2}^{4}\left(\theta_{1} \theta_{2} \theta_{9} \theta_{8} \theta_{10} \theta_{3} \theta_{7}\right)^{4} \\
& +\theta_{6}^{4}\left(\theta_{1} \theta_{9} \theta_{6} \theta_{8} \theta_{10} \theta_{3} \theta_{7}\right)^{4}+\theta_{8}^{4}\left(\theta_{1} \theta_{5} \theta_{2} \theta_{8} \theta_{10} \theta_{3} \theta_{7}\right)^{4}+\theta_{10}^{4}\left(\theta_{5} \theta_{2} \theta_{6} \theta_{8} \theta_{10} \theta_{3} \theta_{7}\right)^{4} \\
& +\theta_{3}^{4}\left(\theta_{5} \theta_{9} \theta_{6} \theta_{8} \theta_{10} \theta_{3} \theta_{7}\right)^{4}+\theta_{7}^{4}\left(\theta_{1} \theta_{5} \theta_{2} \theta_{9} \theta_{6} \theta_{10} \theta_{7}\right)^{4}+\theta_{7}^{4}\left(\theta_{1} \theta_{2} \theta_{9} \theta_{6} \theta_{8} \theta_{3} \theta_{7}\right)^{4} \\
& +\theta_{9}^{4}\left(\theta_{1} \theta_{5} \theta_{2} \theta_{9} \theta_{8} \theta_{10} \theta_{4}\right)^{4}+\theta_{6}^{4}\left(\theta_{1} \theta_{5} \theta_{2} \theta_{6} \theta_{8} \theta_{10} \theta_{4}\right)^{4}+\theta_{2}^{4}\left(\theta_{1} \theta_{5} \theta_{2} \theta_{9} \theta_{8} \theta_{3} \theta_{4}\right)^{4} \\
& +\theta_{6}^{4}\left(\theta_{1} \theta_{5} \theta_{9} \theta_{6} \theta_{8} \theta_{3} \theta_{4}\right)^{4}+\theta_{1}^{4}\left(\theta_{1} \theta_{5} \theta_{9} \theta_{6} \theta_{10} \theta_{3} \theta_{4}\right)^{4}+\theta_{2}^{4}\left(\theta_{5} \theta_{2} \theta_{9} \theta_{6} \theta_{10} \theta_{3} \theta_{4}\right)^{4} \\
& +\theta_{1}^{4}\left(\theta_{1} \theta_{2} \theta_{6} \theta_{8} \theta_{10} \theta_{3} \theta_{4}\right)^{4}+\theta_{5}^{4}\left(\theta_{5} \theta_{2} \theta_{6} \theta_{8} \theta_{10} \theta_{3} \theta_{4}\right)^{4}+\theta_{9}^{4}\left(\theta_{2} \theta_{9} \theta_{6} \theta_{8} \theta_{10} \theta_{3} \theta_{4}\right)^{4} \\
& +\theta_{8}^{4}\left(\theta_{5} \theta_{9} \theta_{6} \theta_{8} \theta_{10} \theta_{3} \theta_{4}\right)^{4}+\theta_{10}^{4}\left(\theta_{1} \theta_{5} \theta_{9} \theta_{8} \theta_{10} \theta_{3} \theta_{4}\right)^{4}+\theta_{3}^{4}\left(\theta_{1} \theta_{5} \theta_{2} \theta_{8} \theta_{10} \theta_{3} \theta_{4}\right)^{4} \\
& +\theta_{5}^{4}\left(\theta_{1} \theta_{5} \theta_{2} \theta_{9} \theta_{6} \theta_{7} \theta_{4}\right)^{4}+\theta_{2}^{4}\left(\theta_{1} \theta_{5} \theta_{2} \theta_{6} \theta_{8} \theta_{7} \theta_{4}\right)^{4}+\theta_{9}^{4}\left(\theta_{1} \theta_{5} \theta_{9} \theta_{6} \theta_{8} \theta_{7} \theta_{4}\right)^{4} \\
& +\theta_{8}^{4}\left(\theta_{1} \theta_{2} \theta_{9} \theta_{6} \theta_{8} \theta_{7} \theta_{4}\right)^{4}+\theta_{1}^{4}\left(\theta_{1} \theta_{2} \theta_{9} \theta_{8} \theta_{10} \theta_{7} \theta_{4}\right)^{4}+\theta_{5}^{4}\left(\theta_{5} \theta_{2} \theta_{9} \theta_{8} \theta_{10} \theta_{7} \theta_{4}\right)^{4} \\
& +\theta_{6}^{4}\left(\theta_{2} \theta_{9} \theta_{6} \theta_{8} \theta_{10} \theta_{7} \theta_{4}\right)^{4}+\theta_{10}^{4}\left(\theta_{1} \theta_{2} \theta_{9} \theta_{6} \theta_{10} \theta_{7} \theta_{4}\right)^{4}+\theta_{10}^{4}\left(\theta_{1} \theta_{5} \theta_{6} \theta_{8} \theta_{10} \theta_{7} \theta_{4}\right)^{4} \\
& +\theta_{1}^{4}\left(\theta_{1} \theta_{5} \theta_{2} \theta_{9} \theta_{3} \theta_{7} \theta_{4}\right)^{4}+\theta_{6}^{4}\left(\theta_{5} \theta_{2} \theta_{9} \theta_{6} \theta_{3} \theta_{7} \theta_{4}\right)^{4}+\theta_{8}^{4}\left(\theta_{5} \theta_{2} \theta_{9} \theta_{8} \theta_{3} \theta_{7} \theta_{4}\right)^{4} \\
& +\theta_{5}^{4}\left(\theta_{1} \theta_{5} \theta_{6} \theta_{10} \theta_{3} \theta_{7} \theta_{4}\right)^{4}+\theta_{2}^{4}\left(\theta_{1} \theta_{2} \theta_{6} \theta_{10} \theta_{3} \theta_{7} \theta_{4}\right)^{4}+\theta_{9}^{4}\left(\theta_{1} \theta_{9} \theta_{6} \theta_{10} \theta_{3} \theta_{7} \theta_{4}\right)^{4} \\
& +\theta_{8}^{4}\left(\theta_{1} \theta_{6} \theta_{8} \theta_{10} \theta_{3} \theta_{7} \theta_{4}\right)^{4}+\theta_{10}^{4}\left(\theta_{5} \theta_{2} \theta_{9} \theta_{10} \theta_{3} \theta_{7} \theta_{4}\right)^{4}+\theta_{3}^{4}\left(\theta_{1} \theta_{2} \theta_{9} \theta_{6} \theta_{3} \theta_{7} \theta_{4}\right)^{4} \\
& +\theta_{3}^{4}\left(\theta_{1} \theta_{5} \theta_{6} \theta_{8} \theta_{3} \theta_{7} \theta_{4}\right)^{4}+\theta_{3}^{4}\left(\theta_{2} \theta_{9} \theta_{8} \theta_{10} \theta_{3} \theta_{7} \theta_{4}\right)^{4}+\theta_{7}^{4}\left(\theta_{1} \theta_{5} \theta_{2} \theta_{8} \theta_{10} \theta_{7} \theta_{4}\right)^{4} \\
& +\theta_{7}^{4}\left(\theta_{1} \theta_{5} \theta_{9} \theta_{8} \theta_{3} \theta_{7} \theta_{4}\right)^{4}+\theta_{7}^{4}\left(\theta_{5} \theta_{9} \theta_{6} \theta_{10} \theta_{3} \theta_{7} \theta_{4}\right)^{4}+\theta_{7}^{4}\left(\theta_{2} \theta_{6} \theta_{8} \theta_{10} \theta_{3} \theta_{7} \theta_{4}\right)^{4} \\
& +\theta_{4}^{4}\left(\theta_{1} \theta_{5} \theta_{2} \theta_{9} \theta_{6} \theta_{10} \theta_{4}\right)^{4}+\theta_{4}^{4}\left(\theta_{1} \theta_{2} \theta_{9} \theta_{6} \theta_{8} \theta_{3} \theta_{4}\right)^{4}+\theta_{4}^{4}\left(\theta_{5} \theta_{9} \theta_{6} \theta_{8} \theta_{10} \theta_{7} \theta_{4}\right)^{4} \\
& +\theta_{4}^{4}\left(\theta_{5} \theta_{2} \theta_{6} \theta_{8} \theta_{3} \theta_{7} \theta_{4}\right)^{4}+\theta_{4}^{4}\left(\theta_{1} \theta_{5} \theta_{2} \theta_{10} \theta_{3} \theta_{7} \theta_{4}\right)^{4}+\theta_{4}^{4}\left(\theta_{1} \theta_{9} \theta_{8} \theta_{10} \theta_{3} \theta_{7} \theta_{4}\right)^{4} \text {. }
\end{aligned}
$$

Then we get four invariants $I_{2}, I_{4}, I_{6}, I_{10}$ of weight $2,4,6$ and 10 :

$$
I_{2}:=\frac{h_{12}}{h_{10}}, \quad I_{4}:=h_{4}, \quad I_{6}:=\frac{h_{16}}{h_{10}}, \quad I_{10}:=h_{10}
$$

From these invariants we can deduce absolute invariants $j_{1}, j_{2}$ and $j_{3}$. The three $j$ invariants are rational generators of the field of absolute invariants. Two principally polarized abelian varieties of dimension two are isomorphic if and only if they have the same $j$-invariants [5]. These are given by

$$
j_{1}:=\frac{I_{2}^{5}}{I_{10}}, \quad j_{2}:=\frac{I_{4} I_{2}^{3}}{I_{10}}, \quad j_{3}:=\frac{I_{6} I_{2}^{2}}{I_{10}} .
$$

\section{From Igusa's invariants to Mestre's invariants}

Since the absolute Igusa invariants $j_{1}, j_{2}, j_{3}$ are rational generators of the field of invariants, we can write the invariants for Mestre's algorithm in terms of them. Mestre gives formulae (p. 319 of [12]) which make it possible to express the coefficients of the conic and the cubic. We follow Mestre's notation.

Set

$$
j_{1}^{\prime}=\frac{A^{5}}{D}, \quad j_{2}^{\prime}=\frac{A^{3} B}{D}, \quad j_{3}^{\prime}=\frac{A^{2} C}{D}
$$


where $A, B, C, D$ are invariants of degree 2, 4, 6 and 10 given by Mestre. Note that $A, B, C, D$ are not the same as the Igusa invariants $I_{2}, I_{4}, I_{6}, \Delta$. The absolute invariants $j_{1}^{\prime}, j_{2}^{\prime}, j_{3}^{\prime}$ are also rational generators of $\mathbb{Q}\left(j_{1}, j_{2}, j_{3}\right)$.

We obtain the following transformation formulae:

$j_{1}^{\prime}=-\frac{j_{1}}{120^{5}}, \quad j_{2}^{\prime}=\frac{720 j_{1}^{\prime}}{6750}-\frac{j_{2}}{120^{3} \cdot 6750}, \quad j_{3}^{\prime}=\frac{j_{3}}{120^{2} \cdot 2025100}+\frac{1080 j_{2}^{\prime}}{2025}-\frac{16 j_{1}^{\prime}}{375}$.

Since $\alpha=\frac{D}{\Delta}$ is an absolute invariant, we can find an expression for it in terms of the $j_{i}^{\prime}$ :

$$
\alpha=-\frac{1}{4556250}\left(\frac{1}{j_{1}^{\prime}}+62208\right)+\frac{16 j_{2}^{\prime}}{75 j_{1}^{\prime}}+\frac{16 j_{3}^{\prime}}{45 j_{1}^{\prime}}-2 \frac{j_{2}^{\prime 2}}{3 j_{1}^{\prime 2}}-\frac{4 j_{2}^{\prime} j_{3}^{\prime}}{3 j_{1}^{\prime 2}} .
$$

We normalize Mestre's invariants $Q_{i j}$ and $H_{l_{1}, \ldots, l_{3}}$ in such a way that they become absolute invariants. For simplicity denote $j_{i}^{\prime}$ by $j_{i}$. Then

$$
\begin{aligned}
& Q_{11}^{\prime}=\frac{Q_{11}}{A^{3}}=\frac{\left(2 j_{3}+\frac{1}{3} j_{2}\right)}{j_{1}}, \\
& Q_{12}^{\prime}=\frac{Q_{12}}{A^{4}}=\frac{2}{3} \frac{j_{2}^{2}+j_{1} j_{3}}{j_{1}^{2}}, \\
& Q_{13}^{\prime}=Q_{22}^{\prime}=\frac{Q_{13}}{A^{5}}=\alpha, \\
& Q_{23}^{\prime}=\frac{Q_{23}}{A^{6}}=\frac{1}{j_{1}^{2}}\left(\frac{j_{2}^{3}}{3 j_{1}}+\frac{4 j_{2} j_{3}}{9}+\frac{2 j_{3}^{2}}{3}\right) \text {, } \\
& Q_{33}^{\prime}=\frac{Q_{33}}{A^{\top}}=\frac{1}{j_{1}^{2}}\left(\frac{j_{1} j_{2} \alpha}{2}+\frac{2 j_{2}^{2} j_{3}}{9 j_{1}}+\frac{2 j_{3}^{2}}{9}\right), \\
& H_{111}^{\prime}=\frac{H_{111}}{A^{5}}=\frac{2}{9} \frac{j_{1}^{2} j_{3}-6 j_{1} j_{2} j_{3}+9 j_{1}^{2}}{j_{1}^{2}}, \\
& H_{112}^{\prime}=\frac{H_{112}}{A^{6}}=\frac{1}{9} \frac{2 j_{2}^{3}+4 j_{1} j_{2} j_{3}+12 j_{1} j_{3}^{2}+3 j_{1}^{2}}{j_{1}^{3}}, \\
& H_{113}^{\prime}=H_{122}^{\prime}=\frac{H_{113}}{A^{7}}=\frac{1}{9} \frac{j_{2}^{3}+4 / 3 j_{1} j_{2} j_{3}+4 j_{2}^{2} j_{3}+6 j_{1} j_{3}^{2}+3 j_{1} j_{2}}{j_{1}^{3}}, \\
& H_{123}^{\prime}=\frac{H_{123}}{A^{6}}=\frac{1}{18 j_{1}^{3}} 2 \frac{j_{2}^{4}}{j_{1}}+4 j_{2}^{2} j_{3}+\frac{4 j_{1} j_{3}^{2}}{3}+4 j_{2} j_{3}^{2}+3 j_{1} j_{2}+12 j_{1} j_{3}, \\
& H_{133}^{\prime}=\frac{H_{133}}{A^{7}}=\frac{1}{18 j_{1}^{3}}\left(\frac{j_{2}^{4}}{j_{1}}+\frac{4 j_{2}^{2} j_{3}}{3}+\frac{16 j_{2}^{3} j_{3}}{3 j_{1}}+\frac{26 j_{2} j_{3}^{2}}{3}+8 j_{3}^{3}+3 j_{2}^{2}+2 j_{1} j_{3}\right), \\
& H_{222}^{\prime}=\frac{H_{222}}{A^{6}}=\frac{1}{9 j_{1}^{3}}\left(3 \frac{j_{2}^{4}}{j_{1}}+6 j_{2}^{2} j_{3}+\frac{8}{3} j_{1} j_{3}^{2}+2 j_{2} j_{3}^{2}-3 j_{1} j_{3}\right), \\
& H_{223}^{\prime}=\frac{H_{223}}{A^{7}}=\frac{1}{18 j_{1}^{3}}\left(-\frac{2 j_{2}^{3} j_{3}}{3 j_{1}}-\frac{4 j_{2} j_{3}^{2}}{3}-4 j_{3}^{3}+9 j_{2}^{2}+8 j_{1} j_{3}\right), \\
& H_{233}^{\prime}=\frac{H_{233}}{A^{8}}=\frac{1}{18 j_{1}^{3}}\left(\frac{j_{2}^{5}}{j_{1}^{2}}+2 \frac{j_{2}^{3} j_{3}}{j_{1}}+\frac{8}{9} j_{2} j_{3}^{2}+\frac{2 j_{2}^{2} j_{3}^{2}}{3 j_{1}}-j_{2} j_{3}+9 j_{1}\right) \text {, } \\
& H_{333}^{\prime}=\frac{H_{333}}{A^{9}}=\frac{1}{36 j_{1}^{3}}\left(-2 \frac{j_{2}^{4} j_{3}}{j_{1}^{2}}-4 \frac{j_{2}^{2} j_{3}^{2}}{j_{1}}-\frac{16}{9} j_{3}^{3}-\frac{4 j_{2} j_{3}^{3}}{j_{1}}+9 \frac{j_{2}^{3}}{j_{1}}+12 j_{2} j_{3}+20 j_{3}^{2}\right) \text {. }
\end{aligned}
$$

From now on, we write $Q_{i j}$ and $H_{i j k}$ instead of $Q_{i j}^{\prime}$ and $H_{i j k}^{\prime}$.

\section{Mestre's ALGORITHM FOR Finite PRIME FIELDS}

For the idea of the algorithm and its correctness for an arbitrary field $K$, see Mestre's article [12.

We give here an explicit formulation if $K$ is a finite prime field.

Suppose we have the three $j$-invariants $\tilde{j}_{1}, \tilde{j}_{2}, \tilde{j}_{3}$ modulo $p$. Then we get Mestre's invariants

$$
Q_{11}, Q_{12}, Q_{13}=Q_{22}, Q_{23}, Q_{33}
$$


and

$$
H_{111}, H_{112}, H_{113}, H_{123}, H_{133}, H_{222}, H_{223}, H_{233}, H_{333} \text {, }
$$

which are the coefficients of a conic

$$
\sum_{1 \leq i \leq j \leq 3} Q_{i j} x_{i} x_{j}
$$

resp. a cubic

$$
\sum_{1 \leq i \leq j \leq k \leq 3} H_{i j k} x_{i} x_{j} x_{k} .
$$

We would like to parametrize the conic so that the set of solutions is given by

$$
\left(f_{1}(t), f_{2}(t), f_{3}(t)\right) \text {. }
$$

For this we transform the conic into a normal form

$$
Q_{11}^{\prime} x_{1}^{2}+Q_{22}^{\prime} x_{2}^{2}+Q_{33}^{\prime} x_{3}^{2}
$$

We can then parametrize the new conic $Q^{\prime}$ and finally get a parametrization $\left(f_{1}(t), f_{2}(t), f_{3}(t)\right)$ of the conic $Q$ itself.

We plug the solution into the cubic

$$
\sum_{i \leq j \leq k} H_{i j k} f_{i}(t) f_{j}(t) f_{k}(t)
$$

to get the model of the hyperelliptic curve

$$
y^{2}=\sum_{i \leq j \leq k} H_{i j k} f_{i}(t) f_{j}(t) f_{k}(t)=: f(t) .
$$

The polynomial $f(t)$ has degree six. Suppose we would like to apply Cantor's algorithm. Then we need a monic polynomial of degree 5 . The hyperelliptic function field given by

$$
C_{f}: y^{2}=f(t), \quad \operatorname{deg} f=6,
$$

has a model defined over $\mathbb{F}_{p}$

$$
C_{g}: y^{2}=g(t), \quad \operatorname{deg} g=5,
$$

if and only if the curve $C_{f}$ has an $\mathbb{F}_{p}$-rational Weierstrass point. Simply speaking this means that $f(t)$ has a zero in $\mathbb{F}_{p}$. By a projective transformation we move the $\mathbb{F}_{p}$-rational Weierstrass point to infinity and obtain a polynomial of degree five.

We can compute the probability that a polynomial of degree 6 whose discriminant is not equal to zero has an $\mathbb{F}_{p}$-rational point. It is given by

$$
\frac{-24 p-3 p^{3}+10 p^{2}+91 p^{6}+43 p^{4}-117 p^{5}}{144\left(p^{6}-p^{5}\right)} .
$$

The complete algorithm is now given as follows:

\section{Computing the curve from its $j$-invariants}

Input: $j_{1}, j_{2}, j_{3} \in \mathbb{F}_{p}$

Output: A hyperelliptic curve of the form $y^{2}=f(t)$, where $f(t) \in \mathbb{F}_{p}[t], \operatorname{deg}(f)=$ 5 or 6.

1: Compute Mestre's invariants $Q_{i j}, H_{i j k}$ from the $j$-invariants $j_{i}, i=1,2,3$.

2: Parametrize the conic $\left(Q_{i j}\right)$ by $f_{1}(t), f_{2}(t), f_{3}(t)$.

3: Obtain $f(t):=\sum_{i \leq j \leq k} H_{i j k} f_{i}(t) f_{j}(t) f_{k}(t) \in \mathbb{F}_{p}[t]$. 
4: Transform $f$ into a polynomial of degree 5 if possible.

5: Return $y^{2}=f(t)$.

\section{Good Weil numbers}

In this section we describe how to find a suitable prime $p$ such that

$$
w \bar{w}=p,
$$

for $w \in \mathcal{O}_{K}$. The equation (3) is a relative norm equation with respect to the relative field extension $K / K_{0}$. Thus we could choose random primes and try to solve the relative norm equation by applying the function $<$ bnfisintnorm $>$ provided by the PARI library to $p^{2}$. The primes so found are of the most general form, but the method needs heavier machinery (e.g., class groups) and is more difficult to implement than the algorithm we discuss in this section. Our method finds only two possibilities for the group order. For applications this is sufficient.

We assume that the Frobenius $w$ is an element in $\mathcal{O}=\mathcal{O}_{K_{0}}+\eta \mathcal{O}_{K_{0}}$, where $\eta=i \sqrt{a+b \sqrt{d}}$, resp. $\eta=i \sqrt{a+b\left(\frac{-1+\sqrt{d}}{2}\right)}$. The order $\mathcal{O}$ might not be the maximal order $\mathcal{O}_{K}$. Let $D$ be the discriminant of $K_{0}$.

First we consider $D \equiv 0 \bmod 4$. Set $p=w \bar{w}$ and

$$
w=c_{1}+c_{2} \sqrt{d}+\left(c_{3}+c_{4} \sqrt{d}\right) i \sqrt{a+b \sqrt{d}} .
$$

We obtain two equations for $c_{1}, c_{2}, c_{3}$ und $c_{4}$ :

$$
\begin{aligned}
c_{1}^{2}+c_{2}^{2} d+c_{3}^{2} a+c_{4}^{2} a d+2 c_{3} c_{4} b d & =p, \\
2 c_{1} c_{2}+2 c_{3} c_{4} a+c_{3}^{2} b+c_{4}^{2} b d & =0 .
\end{aligned}
$$

From this we deduce the following algorithm:

Good Weil numbers, $D \equiv 0 \bmod 4$

Input: CM-field $K=\mathbb{Q}(i \sqrt{a+b \sqrt{d}})$.

Output: A prime $p$ that satisfies a relative norm equation and the two possible group orders.

1: Choose $c_{3}, c_{4}$ at random $1\left(c_{3}, c_{4}\right)=1$.

2: if $c_{3}^{2} b-c_{4}^{2} d b \not \equiv 0 \bmod 2$ then

3: $\quad$ Start again.

4: end if

5: Set $2 n:=-2 c_{3} c_{4} a-c_{3}^{2} b-c_{4}^{2} b d$.

6: Set $c_{1}:=q$ where $q \mid n$.

7: $c_{2}:=n / q$

8: $p:=c_{1}^{2}+c_{2}^{2} d+c_{3}^{2} a+c_{4}^{2} d a+2 c_{3} c_{4} b d$;

9: if $p$ is not prime then

10: Start again.

11: end if

12: Set possible group order $(p+1)^{2} \pm 4(p+1) c_{1}+4\left(c_{1}^{2}-c_{2}^{2} d\right)$.

The algorithm for $D \equiv 1 \bmod 4$ is based on the same idea.

\footnotetext{
${ }^{1}$ If $c_{3}, c_{4}$ have approximately $n$ digits then $p$ will have around $2 n$ digits.
} 
Good Weil numbers, $D \equiv 1 \bmod 4$

Input: CM-field $K=\mathbb{Q}\left(i \sqrt{a+b\left(\frac{-1+\sqrt{d}}{2}\right)}\right)$.

Output: A prime $p$ that satisfies a relative norm equation and the two possible group orders.

1: Choose $c_{3}, c_{4}$ at random, $\left(c_{3}, c_{4}\right)=1$.

2: $n:=2 c_{3} c_{4} a-c_{4}^{2} a+c_{3}^{2} b-2 c_{3} c_{4} b+\left(\frac{d+3}{4}\right) b c_{4}^{2}$;

3: if $n \bmod 4 \equiv 2$ then

4: $\quad$ Start again.

5: end if

6: if $n$ even then

7: $\quad c_{2}:=$ even factor of $n$;

8: else

9: $\quad c_{2}:=$ odd factor of $n$;

10: end if

11: $c_{1}:=\frac{1}{2}\left(-\frac{n}{c_{2}}+c_{2}\right)$;

12: $p:=c_{1}^{2}+c_{2}^{2}\left(\frac{d-1}{4}\right)+c_{3}^{2} a+c_{4}^{2} a\left(\frac{d-1}{4}\right)+2 c_{3} c_{4} b\left(\frac{d-1}{4}\right)-b c_{4}^{2}\left(\frac{d-1}{4}\right)$;

13: if $p$ is not prime then

14: $\quad$ Start again.

15: end if

16: Set possible group order $(p+1)^{2} \pm(p+1)\left(4 c_{1}-2 c_{2}\right)+4\left(c_{1}^{2}-c_{1} c_{2}+c_{2}^{2}\left(\frac{1-d}{4}\right)\right)$.

Note that this elementary method needs the factorization of an integer in step 6 . It is not advisable to factor the integer completely. Much faster is a trial factorization up to a fixed bound. Table 1 shows the time (in seconds) to find 1000 primes of size approximately $2^{80}$ satisfying a relative norm equation.

TABLE 1.

\begin{tabular}{|r|l|l|}
\hline CM-field & elementary programm & use of <bnfisintnorm $>$ \\
\hline \hline $\mathbb{Q}(i \sqrt{2+\sqrt{2}})$ & 247 & 296 \\
$\mathbb{Q}(i \sqrt{3+\sqrt{2}})$ & 256 & 371 \\
$\mathbb{Q}(i \sqrt{4+\sqrt{2}})$ & 252 & 394 \\
$\mathbb{Q}(i \sqrt{29+6 \sqrt{23}})$ & 302 & 403 \\
$\mathbb{Q}\left(i \sqrt{\frac{3+\frac{-1+\sqrt{5}}{2}}{2}}\right)$ & 243 & 328 \\
$\mathbb{Q}\left(i \sqrt{4+\frac{-1+\sqrt{5}}{2}}\right)$ & 241 & 424 \\
$\mathbb{Q}\left(i \sqrt{5+\frac{-1+\sqrt{5}}{2}}\right)$ & 240 & 429 \\
$\mathbb{Q}\left(i \sqrt{55+4 \frac{-1+\sqrt{53}}{2}}\right)$ & 285 & 378 \\
$\mathbb{Q}\left(i \sqrt{6+\frac{-1+\sqrt{73}}{2}}\right)$ & 263 & 424 \\
$\mathbb{Q}\left(i \sqrt{6+\frac{-1+\sqrt{89}}{2}}\right)$ & 268 & 197 \\
$\mathbb{Q}\left(i \sqrt{7+\frac{-1+\sqrt{101}}{2}}\right)$ & 257 & 303 \\
$\mathbb{Q}\left(i \sqrt{6+\frac{-1+\sqrt{113}}{2}}\right)$ & 266 & 435 \\
$\mathbb{Q}\left(i \sqrt{9+\frac{-1+\sqrt{141}}{2}}\right)$ & 292 & 356 \\
\hline
\end{tabular}




\section{The COMPLETE ALGORITHM}

Now we are able to give the complete algorithm.

Before starting with the construction of a hyperelliptic curve we have to fix a CM-field, and we have to find a suitable prime field $\mathbb{F}_{p}$.

Precomputations for the group order

1: Choose a CM-field $K=\mathbb{Q}(i \sqrt{a+b \sqrt{d}})$.

2: Find a prime $p$ such that there exists $w \in K$ with $w \bar{w}=p$.

3: Compute the two (resp. four) possible group orders $n_{1}, n_{2}\left(n_{3}, n_{4}\right)$ depending on $p$ and $\mathcal{O}_{K}$.

4: if $n_{1}$ and $n_{2}$ (resp. $n_{3}$ and $n_{4}$ ) have no large prime factor then

5: $\quad$ Go to 2 .

6: else

7: $\quad$ Return $K, p, n_{1}$ and $n_{2}$.

8: end if

Once we have found a CM-field $K$ and a suitable prime $p$, we are left with the task of finding a hyperelliptic curve defined over $\mathbb{F}_{p}$ having complex multiplication by the order $\mathcal{O}_{K}$.

Construction of hyperelliptic curves suitable for cryptography

Input: CM-field $K, h_{K_{0}}=1$, prime $p$ and the two (sometimes four) possible group orders $n_{1}$ and $n_{2}$ (resp. $n_{3}, n_{4}$ ).

Output: A hyperelliptic curve of the form $y^{2}=f(t)$, where $f(t) \in \mathbb{F}_{p}[t], \operatorname{deg}(f)=$ 5 or 6.

1: Choose a complete set (up to isomorphism) of period matrices $\Omega_{i}$ of all simple principally polarized abelian varieties having complex multiplication by $\mathcal{O}_{K}$ (see Section 3). Let $s$ be the size of the set of isomorphism classes.

2: Compute all even theta constants

$$
\theta\left[\begin{array}{l}
\delta \\
\epsilon
\end{array}\right]\left(\Omega_{i}, 0\right)=\sum_{n \in \mathbb{Z}^{2}} \exp \left(\pi i\left(n+\frac{1}{2} \delta\right)^{t} \Omega_{i}\left(n+\frac{1}{2} \delta\right)+2\left(n+\frac{1}{2} \delta\right)^{t}\left(\frac{1}{2} \epsilon\right)\right) .
$$

See Section 4 .

3: Compute the $3 s$ generating $j$-invariants

$$
j_{1}^{(i)}, j_{2}^{(i)}, j_{3}^{(i)}, \quad i=1, \ldots, s .
$$

4: Compute the class polynomials

$$
H_{1}(X)=\prod_{i=1}^{s}\left(X-j_{1}^{(i)}\right), \quad H_{2}(X)=\prod_{i=1}^{s}\left(X-j_{2}^{(i)}\right), \quad H_{3}(X)=\prod_{i=1}^{s}\left(X-j_{3}^{(i)}\right) .
$$

5: Find the denominator (see Remark 9.1) and get the polynomials $H_{i}^{\prime}(X) \in \mathbb{Z}[X]$.

6: for all $\left(a_{1}, a_{2}, a_{3}\right), a_{i}$ zero of $H_{i}^{\prime}(X) \bmod p$ do

7: $\quad$ Set

$$
j_{1}:=a_{1} ; \quad j_{2}:=a_{2} ; \quad j_{3}:=a_{3} .
$$

8: Compute Mestre's invariants (see Section 6).

9: Apply Mestre's algorithm to get a curve

$$
C: y^{2}=f(t), \quad f(t) \in \mathbb{F}_{p}[t], \quad \operatorname{deg} f(t)=6 .
$$

10: $\quad$ if $\# J(C)=n_{1}$ or $n_{2}$ then

11: $\quad$ Return $C$; 
12: $\quad$ end if

13: end for

Remark 9.1. 1. The algorithm is restricted to CM-fields whose real subfield has class number one. This ensures that the ideals in $\mathcal{O}_{K}$ have a relative integer basis with respect to $\mathcal{O}_{K_{0}}$. Therefore it is possible to give period matrices of the principally polarized abelian varieties with complex multiplication by $\mathcal{O}_{K}$ (see Theorem 3.1).

2. For step 5 of the algorithm we applied the continued fraction algorithm to the second highest coefficient of the polynomial to obtain a possible denominator $d_{K, H_{i}(X)}$. This method gives us almost always a polynomial in $\mathbb{Z}$ which is not necessarily primitive.

There exist exceptions, e.g.,

$$
K=\mathbb{Q}\left(i \sqrt{5+\frac{-1+\sqrt{13}}{2}}\right)
$$

where the denominator of the polynomial $H_{1}(X)$ contains an additional power of three, i.e., $3^{2}$.

3. In contrast to elliptic curves, where the class polynomial always splits into linear factors, the class polynomials $H_{i}(X)$ do not have to split into linear factors even when $p$ satisfies a relative norm equation. For the correctness of our method it is only important that $H_{i}(X)$ have a linear factor modulo $p$. This is a consequence from class field theory.

It is even more efficient to take primes which do not split completely, since the for-loop in step 6 takes fewer rounds in this case.

Example 9.2. Consider the CM-field $\mathbb{Q}(i \sqrt{3+\sqrt{2}})$ and the prime

$$
p=907978164842524484436193557995633106029 \text {. }
$$

Each of the class polynomials $H_{1}(X), H_{2}(X)$ and $H_{3}(X)$ splits into one factor of degree two and two factors of degree one.

The two linear factors give us hyperelliptic curves defined over $\mathbb{F}_{p}$, and the irreducible factor of degree two leads to curves defined over $\mathbb{F}_{p^{2}}$.

\section{Complexity of the Algorithm}

10.1. Analysis. The algorithm falls into two parts:

1. Computation of the class polynomial.

2. Mestre's algorithm.

The running time of the first part is dominated by the computation of the theta constants. In Section 4 we saw that the computation of the theta constants depends on the value of the first successive minima of the period matrix. An exact analysis is therefore rather difficult.

However, since we cannot compute up to arbitrarily high precision, the number of CM-fields we consider is restricted (see also Section [13). In principle we could compute all possible class polynomials in advance. 
Table 2 will give an idea about the complexity of the computation of the class polynomials. The first three columns describe the CM-field $K$

$$
\begin{array}{ll}
\mathbb{Q}(i \sqrt{a+b \sqrt{d}}), \quad d=\frac{D}{4}, & \text { if } D \equiv 0 \bmod 4, \\
\mathbb{Q}\left(i \sqrt{a+b \frac{-1+\sqrt{d}}{2}}\right), \quad d=D, & \text { if } D \equiv 1 \bmod 4 .
\end{array}
$$

The fourth column gives the class number of $K$ and the fifth the number of possible polarizations. Note that in all these cases $h_{K}(\# \mathrm{Pol})$ is the degree of the class polynomial.

Recall that the monic class polynomial has rational coefficients. The sixth column gives the number of decimal digits of the denominator of the second highest coefficient of the class polynomial. The seventh column gives the precision which is necessary for the computations to get the right result. The eighth column gives the time in seconds.

Some of the CM-fields are taken from the tables in [15] and [11. We were able to compute the class polynomial of a CM-fields of class number 10 .

The second part is the application of Mestre's algorithm. First we have to factor the class polynomial. There exists an efficient probabilistic algorithm [3] which takes

$$
O\left(n^{2+\epsilon}+n \log p\right)
$$

operations in $\mathbb{F}_{p}$, where $n$ is the degree of the polynomial. In our situation the degree of the polynomial is bounded and small. So we can estimate the number of operations in $\mathbb{F}_{p}$ by $O(\log p)$. Note that we have to apply Mestre's algorithm $s^{3}$ times, where $s$ is the degree of the class polynomial. In most cases $s$ equals $2 h_{K}$. Especially we have to compute $s^{3}$ scalar multiplications on a hyperelliptic Jacobian. Every scalar multiplication takes

$$
O\left(g^{2} \log p\right)
$$

field operations (see 16 for the complexity of a single composition on a hyperelliptic Jacobian). Thus as an overall complexity (once the class polynomial is computed) we get

$$
O\left(\left(2 h_{K}\right)^{3} \log p\right)
$$

operations in $\mathbb{F}_{p}$.

TABLE 2 .

\begin{tabular}{|r|r|l||l|l|l|r|r|}
\hline \multicolumn{3}{|c|}{ CM-field } & & & & & \\
\hline D & $\mathbf{a}$ & $\mathbf{b}$ & $h_{K}$ & $\begin{array}{l}\# \\
\text { pol. }\end{array}$ & $\begin{array}{l}\text { denom. } \\
\text { dec. digits }\end{array}$ & prec. & $\begin{array}{r}\text { time } \\
\text { (in s.) }\end{array}$ \\
\hline 5 & 3 & 1 & 1 & 1 & 1 & 20 & 1 \\
5 & 4 & 1 & 2 & 2 & 11 & 50 & 1 \\
5 & 7 & 1 & 1 & 2 & 1 & 20 & 1 \\
5 & 6 & 2 & 2 & 1 & 13 & 100 & 1 \\
5 & 8 & 1 & 4 & 2 & 23 & 100 & 18 \\
\hline
\end{tabular}


TABle 2. (Continued)

\begin{tabular}{|c|c|c|c|c|c|c|c|}
\hline \multicolumn{3}{|c|}{ CM-field } & \multirow[b]{2}{*}{$h_{K}$} & \multirow[b]{2}{*}{$\begin{array}{l}\# \\
\text { pol. }\end{array}$} & \multirow[b]{2}{*}{$\begin{array}{l}\text { denom. } \\
\text { dec. digits }\end{array}$} & \multirow[b]{2}{*}{ prec. } & \multirow[b]{2}{*}{$\begin{array}{r}\text { time } \\
\text { (in s.) }\end{array}$} \\
\hline D & $\mathbf{a}$ & $\mathbf{b}$ & & & & & \\
\hline 5 & 16 & 7 & 4 & 2 & 59 & 200 & 52 \\
\hline 5 & 23 & 5 & 1 & 2 & 52 & 100 & 3 \\
\hline 5 & 91 & 52 & 2 & 1 & 50 & 150 & 3 \\
\hline 5 & 35 & 8 & 5 & 2 & 29 & 400 & 81 \\
\hline 5 & 18 & 6 & 4 & 1 & 73 & 400 & 35 \\
\hline 8 & 2 & 1 & 1 & 1 & 1 & 20 & 1 \\
\hline 8 & 3 & 1 & 2 & 2 & 3 & 50 & 1 \\
\hline 8 & 6 & 1 & 4 & 2 & 17 & 200 & 23 \\
\hline 8 & 13 & 4 & 2 & 2 & 42 & 200 & 7 \\
\hline 8 & 13 & 6 & 3 & 2 & 4 & 100 & 2 \\
\hline 8 & 15 & 4 & 5 & 2 & 4 & 300 & 71 \\
\hline 8 & 23 & 8 & 7 & 2 & 22 & 500 & 568 \\
\hline 12 & 11 & 4 & 4 & 2 & 4 & 100 & 8 \\
\hline 13 & 3 & 1 & 2 & 2 & 1 & 50 & 1 \\
\hline 13 & 6 & 2 & 2 & 2 & 16 & 150 & 9 \\
\hline 13 & 8 & 3 & 1 & 1 & 1 & 20 & 1 \\
\hline 13 & 16 & 3 & 1 & 2 & 30 & 100 & 1 \\
\hline 17 & 9 & 1 & 4 & 1 & 22 & 100 & 6 \\
\hline 17 & 6 & 1 & 4 & 2 & 38 & 200 & 16 \\
\hline 21 & 5 & 1 & 4 & 2 & 45 & 300 & 51 \\
\hline 24 & 17 & 6 & 6 & 2 & 16 & 600 & 401 \\
\hline 28 & 7 & 2 & 4 & 2 & 12 & 150 & 10 \\
\hline 29 & 7 & 2 & 4 & 2 & 24 & 200 & 31 \\
\hline 29 & 24 & 7 & 4 & 2 & 7 & 150 & 20 \\
\hline 33 & 95 & 28 & 4 & 2 & 40 & 200 & 27 \\
\hline 37 & 19 & 5 & 3 & 2 & 9 & 100 & 16 \\
\hline 37 & 26 & 7 & 3 & 2 & 33 & 200 & 90 \\
\hline 41 & 10 & 2 & 4 & 2 & 8 & 150 & 14 \\
\hline 44 & 67 & 20 & 4 & 2 & 22 & 200 & 26 \\
\hline 53 & 55 & 4 & 1 & 1 & 33 & 100 & 1 \\
\hline 56 & 53 & 14 & 2 & 2 & 33 & 100 & 3 \\
\hline 57 & 9 & 2 & 2 & 2 & 6 & 100 & 3 \\
\hline 61 & 9 & 2 & 2 & 2 & 30 & 100 & 2 \\
\hline 69 & 5 & 1 & 4 & 2 & 21 & 200 & 292 \\
\hline 73 & 6 & 1 & 4 & 2 & 13 & 150 & 20 \\
\hline 73 & 5 & 1 & 1 & 2 & 11 & 50 & 1 \\
\hline 76 & 279 & 64 & 4 & 2 & 8 & 150 & 152 \\
\hline 88 & 5 & 1 & 2 & 2 & 37 & 150 & 4 \\
\hline 89 & 11 & 2 & 4 & 2 & 25 & 300 & 83 \\
\hline 92 & 29 & 6 & 4 & 2 & 26 & 200 & 216 \\
\hline 92 & 77 & 16 & 2 & 2 & 31 & 200 & 20 \\
\hline 97 & 8 & 1 & 3 & 2 & 11 & 100 & 8 \\
\hline 101 & 7 & 1 & 3 & 2 & 1 & 100 & 7 \\
\hline 109 & 9 & 1 & 1 & 2 & 5 & 20 & 1 \\
\hline 124 & 39 & 7 & 4 & 2 & 40 & 200 & 310 \\
\hline 124 & 23 & 4 & 2 & 2 & 19 & 150 & 3 \\
\hline 129 & 13 & 2 & 4 & 2 & 37 & 200 & 82 \\
\hline 137 & 10 & 1 & 4 & 2 & 33 & 300 & 532 \\
\hline 141 & 9 & 1 & 2 & 2 & 35 & 300 & 44 \\
\hline 233 & 10 & 1 & 1 & 2 & 27 & 50 & 1 \\
\hline 389 & 114 & 11 & 1 & 2 & 50 & 100 & 2 \\
\hline
\end{tabular}


10.2. Notes on implementation. The two parts of the algorithm differ not only in the complexity but also in the difficulty of the implementation.

For the first part we need a library that supports computations in relative number fields. Most suitable for this task is the C-library PARI (ftp://megrez.math.ubordeaux.fr/pub/pari/).

The second part is easier to implement. It requires efficient polynomial arithmetic. We used the library NTL written in C++ (http://www. shoup.net/ntl/).

\section{Statistics}

11.1. Primes interesting for implementations. Some primes are especially well suited for implementions (see [6], 20]). In Table 3 we give a list of Mersenne primes and generalized Mersenne primes. We tested whether they split in a given CM-field or not, and whether the corresponding group order contains a large prime factor.

\section{TABLE 3 .}

\begin{tabular}{|l|l|l||l||r|}
\hline$D$ & $a$ & $b$ & prime & $k$, group order equals $k \cdot q, q$ prime \\
\hline 5 & 6 & 2 & $2^{89}-1$ & 580 \\
& & & $2^{130}-5$ & 4 \\
\hline 5 & 16 & 7 & $2^{150}-3$ & 64 \\
\hline 5 & 18 & 7 & $2^{192}-2^{64}-1$ & 1 \\
& & & $2^{224}-2^{96}+1$ & 1 \\
\hline 5 & 11 & 1 & $2^{96}-2^{32}+1$ & 151 \\
& & & $2^{116}-3$ & 59 \\
& & & $2^{113}-3$ & 11 \\
\hline 5 & 19 & 4 & $2^{107}-1$ & 16 \\
\hline 5 & 23 & 5 & $2^{224}-2^{96}+1$ & 109 \\
& & & $2^{127}-1$ & 1 \\
\hline 8 & 5 & 2 & $2^{89}-1$ & 188 \\
\hline 8 & 6 & 3 & $2^{96}-2^{32}+1$ & 32 \\
\hline 8 & 13 & 6 & $2^{116}-3$ & 4 \\
\hline 8 & 19 & 8 & $2^{213}-3$ & 167 \\
\hline 13 & 5 & 1 & $2^{192}-2^{64}-1$ & 816 \\
\hline 13 & 6 & 2 & $2^{150}-3$ & 16 \\
\hline 13 & 11 & 4 & $2^{116}-3$ & 13 \\
\hline 13 & 75 & 20 & $2^{127}-1$ & 859 \\
\hline 17 & 3 & 1 & $2^{107}-1$ & 64 \\
\hline 17 & 27 & 8 & $2^{107}-1$ & 300 \\
\hline 21 & 15 & 4 & $2^{118}-3$ & 647 \\
\hline 24 & 3 & 1 & $2^{174}-3$ & 747 \\
\hline 28 & 7 & 2 & $2^{127}-1$ & 511 \\
\hline 29 & 15 & 4 & $2^{107}-1$ & 1 \\
\hline 37 & 19 & 5 & $2^{107}-1$ & 67 \\
\hline 37 & 26 & 7 & $2^{96}-2^{32}+1$ & \\
\hline
\end{tabular}


TABle 3. (Continued)

\begin{tabular}{|l|l|l||l|r|}
\hline$D$ & $a$ & $b$ & prime & $k$, group order equals $k \cdot q, q$ prime \\
\hline 41 & 6 & 1 & $2^{174}-3$ & 100 \\
\hline 44 & 4 & 1 & $2^{94}-3$ & 16 \\
& & & $2^{174}-3$ & 280 \\
\hline 44 & 7 & 2 & $2^{94}-3$ & 14 \\
\hline 53 & 7 & 1 & $2^{192}-2^{64}-1$ & 1 \\
\hline 61 & 67 & 12 & $2^{94}-3$ & 25 \\
\hline 89 & 6 & 1 & $2^{127}-1$ & 136 \\
\hline 129 & 13 & 2 & $2^{96}-2^{32}+1$ & 20 \\
\hline 149 & 7 & 1 & $2^{192}-2^{64}-1$ & 7 \\
\hline 269 & 9 & 1 & $2^{94}-3$ & 16 \\
\hline
\end{tabular}

11.2. Probability of the group order's being almost prime. For every CMfield given in Table 4 we chose 5001 primes of the size $2^{80}$ that satisfy a relative norm equation, and determined the two corresponding group orders.

We counted the number of group orders which are of the form

$$
k \cdot q_{\text {prime }}
$$

where $k=1, \ldots, 9, k \leq 1000$ and $k>1000$. The first column gives the parameter $D, a, b$ of the CM-field.

The CM-field imposes some divisibility conditions on the group order. In the case where $\mathcal{O}_{K}=\mathcal{O}_{K_{0}}+\eta \mathcal{O}_{K_{0}}$ with a purely imaginary element $\eta \in \mathcal{O}_{K}$, the group order can easily be seen to be a multiple of 4 .

It is possible to generalize the heuristics from [7, p. 162, on the probability of prime group order to hyperelliptic curves with complex multiplication. This requires more theoretical background, and will be covered by the author in a forthcoming paper.

\section{TABLE 4.}

\begin{tabular}{|l|l|l|l|l|l|l|l|l|l|l|l|l|}
\hline CM-field & 1 & 2 & 3 & 4 & 5 & 6 & 7 & 8 & 9 & 10 & $11-1000$ & $>1000$ \\
& & & & & & & & & & & & \\
\hline $5,6,2$ & 0 & 0 & 0 & 130 & 0 & 0 & 0 & 0 & 0 & 0 & 431 & 9439 \\
$5,35,8$ & 135 & 0 & 0 & 0 & 94 & 0 & 0 & 0 & 0 & 0 & 421 & 9350 \\
$8,3,1$ & 0 & 0 & 0 & 0 & 0 & 0 & 0 & 93 & 0 & 0 & 374 & 9533 \\
$8,5,2$ & 0 & 0 & 0 & 147 & 0 & 0 & 0 & 0 & 0 & 0 & 431 & 9424 \\
$8,6,1$ & 0 & 0 & 0 & 149 & 0 & 0 & 0 & 0 & 0 & 0 & 438 & 9458 \\
$13,3,1$ & 0 & 0 & 0 & 52 & 0 & 0 & 0 & 0 & 0 & 0 & 365 & 9583 \\
$13,8,3$ & 47 & 0 & 0 & 0 & 0 & 0 & 0 & 0 & 106 & 0 & 397 & 9450 \\
$13,5,1$ & 55 & 0 & 99 & & 0 & 0 & 0 & 0 & 75 & 0 & 360 & 9411 \\
$13,7,1$ & 0 & 0 & 0 & 62 & 0 & 0 & 0 & 0 & 0 & 0 & 407 & 9531 \\
$13,75,20$ & 170 & 0 & 0 & 0 & 0 & 0 & 0 & 0 & 37 & 0 & 448 & 9345 \\
$13,16,6$ & 0 & 0 & 0 & 237 & 0 & 0 & 0 & 0 & 0 & 0 & 464 & 9299 \\
$13,11,4$ & 57 & 0 & 90 & 0 & 0 & 0 & 0 & 0 & 71 & 0 & 378 & 9404 \\
$17,11,4$ & 0 & 0 & 0 & 60 & 0 & 0 & 0 & 67 & 0 & 0 & 427 & 9446 \\
$17,6,1$ & 0 & 0 & 0 & 0 & 0 & 0 & 0 & 108 & 0 & 0 & 436 & 9458 \\
$17,147,56$ & 183 & 0 & 0 & 139 & 0 & 0 & 0 & 0 & 0 & 0 & 441 & 9237 \\
\hline
\end{tabular}


TABle 4. (Continued)

\begin{tabular}{|l|l|l|l|l|l|l|l|l|l|l|l|l|}
\hline CM-field & 1 & 2 & 3 & 4 & 5 & 6 & 7 & 8 & 9 & 10 & $11-1000$ & $>1000$ \\
& & & & & & & & & & & & \\
\hline $21,5,1$ & 0 & 0 & 0 & 59 & 0 & 0 & 0 & 0 & 0 & 0 & 423 & 9520 \\
$21,7,1$ & 34 & 0 & 52 & 0 & 24 & 0 & 11 & 0 & 42 & 0 & 394 & 9443 \\
$21,22,7$ & 60 & 0 & 81 & 0 & 15 & 0 & 0 & 0 & 85 & 0 & 369 & 9390 \\
$21,15,4$ & 112 & 0 & 0 & 0 & 78 & 0 & 39 & 0 & 9 & 0 & 395 & 9367 \\
$28,7,2$ & 0 & 33 & 0 & 24 & 0 & 24 & 0 & 14 & 0 & 0 & 421 & 9484 \\
$29,7,2$ & 0 & 0 & 0 & 55 & 0 & 0 & 0 & 0 & 0 & 0 & 328 & 9617 \\
$29,5,1$ & 92 & 0 & 0 & 0 & 51 & 0 & 31 & 0 & 0 & 14 & 441 & 9373 \\
$29,17,5$ & 160 & 0 & 0 & 0 & 0 & 0 & 105 & 0 & 0 & 0 & 406 & 9329 \\
$29,24,7$ & 70 & 0 & 0 & 0 & 50 & 0 & 55 & 0 & 0 & 0 & 392 & 9433 \\
$29,12,3$ & 76 & 0 & 0 & 0 & 51 & 0 & 31 & 0 & 7 & 0 & 390 & 9447 \\
$29,4,1$ & 0 & 0 & 0 & 163 & 0 & 0 & 0 & 0 & 0 & 0 & 405 & 9434 \\
$33,7,2$ & 0 & 0 & 0 & 0 & 0 & 0 & 0 & 116 & 0 & 0 & 325 & 9559 \\
$33,95,28$ & 0 & 0 & 0 & 31 & 0 & 0 & 0 & 41 & 0 & 0 & 392 & 9538 \\
$37,43,12$ & 134 & 0 & 0 & 0 & 0 & 0 & 97 & 0 & 36 & 0 & 401 & 9332 \\
$44,67,20$ & 0 & 0 & 0 & 125 & 0 & 0 & 0 & 0 & 0 & 0 & 383 & 9492 \\
\hline
\end{tabular}

\section{EXAMPLES}

12.1. Complex multiplication by $\mathbb{Q}\left(i \sqrt{8+\frac{-1+\sqrt{5}}{2}}\right)$. We would like to construct a hyperelliptic curve whose Jacobian has complex multiplication by

$$
K=\mathbb{Q}\left(i \sqrt{8+\frac{-1+\sqrt{5}}{2}}\right) .
$$

Note that $K$ is not Galois and has class number four. The fundamental unit of the real quadratic subfield $K_{0}=\mathbb{Q}(\sqrt{5})$ has negative norm.

A relative integral basis for the elements in the class group is given by

$$
\begin{aligned}
& \mathfrak{A}_{1}=\mathcal{O}_{K}=\mathcal{O}_{K_{0}}+\eta \mathcal{O}_{K_{0}}, \\
& \mathfrak{A}_{2}=\left(-3+\left(\frac{-1+\sqrt{5}}{2}\right) \eta\right) \mathcal{O}_{K_{0}}+\eta \mathcal{O}_{K_{0}}, \\
& \mathfrak{A}_{3}=\left(-2+\left(1+\frac{-1+\sqrt{5}}{2}\right) \eta\right) \mathcal{O}_{K_{0}}+\eta \mathcal{O}_{K_{0}}, \\
& \mathfrak{A}_{4}=\left(-6+\left(3+\frac{-1+\sqrt{5}}{2}\right) \eta\right) \mathcal{O}_{K_{0}}+\eta \mathcal{O}_{K_{0}},
\end{aligned}
$$

where

$$
\eta=i \sqrt{8+\frac{-1+\sqrt{5}}{2}} .
$$

We get a representation system of eight principally polarized abelian varieties of dimension two. For each period matrix we compute the theta constants and the invariants. All computations are done with a precision of 300 decimal digits.

We obtain the class polynomials $H_{1}(X), H_{2}(X), H_{3}(X)$. The three denominators are

$$
3^{5} \cdot 11^{6} \cdot 13^{12}, \quad 3^{3} \cdot 11^{4} \cdot 13^{8}, \quad 3^{4} \cdot 11^{4} \cdot 13^{8} .
$$


We find the polynomials in $\mathbb{Z}[x]$ (due to the size of the coefficients of the first class polynomial we resrict ourselves to giving only the second and third polynomial):

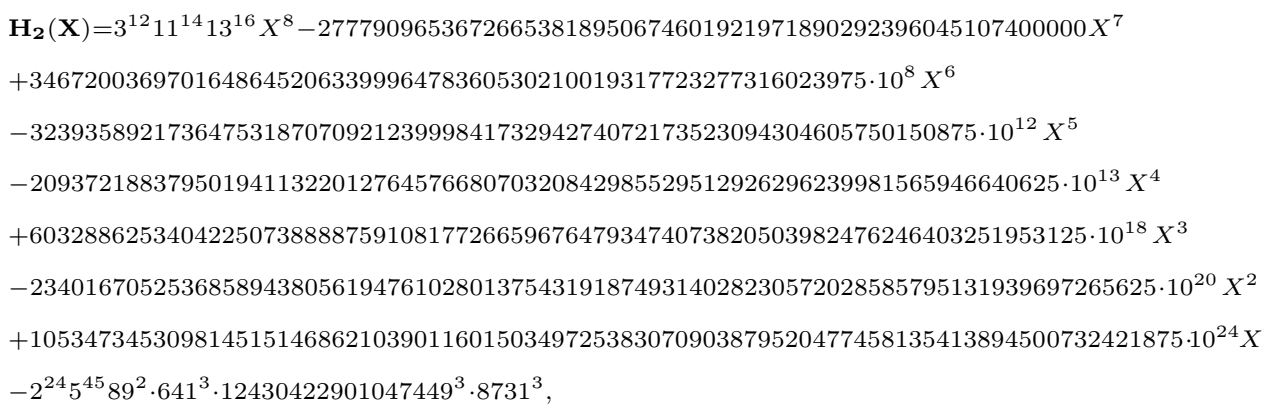

A suitable prime is given by $p=129899216730745422747379980509$. It will give us the two possible group orders:

16873806507261171556624961017693968657279916616235023963476

and

16873806507261171553245686803138166610780248276948805535516.

Note that

$$
\begin{aligned}
n & =16873806507261171556624961017693968657279916616235023963476 \\
& =4 \cdot q_{\text {prime }},
\end{aligned}
$$

where $q_{\text {prime }}$ is a prime number.

Applying Mestre's algorithm, we find the following curve $C$ over $\mathbb{F}_{p}$ :

$$
\begin{aligned}
C: y^{2}=t^{5} & +110854065858994061391078560211 t^{4} \\
& +52690279977948565928475983553 t^{3} \\
& +81016668528840831602117585943 t^{2} \\
& +43842353021798749401773327333 t \\
& +84554758087342364918400819975 .
\end{aligned}
$$

The Jacobian of $C$ has exactly $n$ elements. 
12.2. Complex multiplication by $\mathbb{Q}(i \sqrt{3+\sqrt{7}})$. We consider the CM-field $\mathbb{Q}(i \sqrt{3+\sqrt{7}})$, which has class number two and two polarizations.

The class polynomials $H_{1}(X), H_{2}(X)$ and $H_{3}(X)$ are given by

$$
\begin{gathered}
\mathbf{H}_{1}(\mathbf{X})=X^{4}-2130771672 X^{3}+198502979432505408 X^{2} \\
+6728724103294347293933568 X-5302179309170499300715659264 \\
\mathbf{H}_{\mathbf{2}}(\mathbf{X})=4 X^{4}-236549430 X^{3}+1322300792925225 X^{2} \\
-1088981406809175672000 X-630204755989268223360000 \\
\mathbf{H}_{\mathbf{3}}(\mathbf{X})=64 X^{4}-1045893528 X^{3}+1368605510700597 X^{2} \\
+
\end{gathered}
$$

A suitable prime is given by $p=580943314814642181310688596463593$. It will give us the two possible group orders:

$n_{1}=337495135027824453733283789094149750817279621391373902756574895952$,

$n_{2}=337495135027824453733281165453395182011292833807497899521740278848$.

Note that

$$
n_{1}=16 \cdot q_{\text {prime }},
$$

where $q_{\text {prime }}$ is a prime number.

The curve which corresponds to the group order $n$ is given by

$$
\begin{aligned}
C: y^{2}=t^{5} & +474727596586211034284401845850785 t^{4} \\
& +314748234596474418739580339957648 t^{3} \\
& +314740766532984346191929527993409 t^{2} \\
& +574397988361190658944043563780018 t \\
& +546228693859470379418770593594687
\end{aligned}
$$

\section{Discussion}

In this section we discuss some further improvements and generalizations of the CM-method, as well as the computational limits.

First we would like to mention an idea of van Wamelen to speed up the computation of the theta constants. He suggests in 22 to apply generators of the group $S p_{2}(\mathbb{Z})$ to the period matrix $\Omega_{i}$ in order to maximize the first successive minima of $\Omega_{i}$.

We would like to mention that our method does not work for fields of small characteristic. Let $C$ be a hyperelliptic curve defined over a finite field whose absolute invariants lie in $\mathbb{F}_{q}$ where $q=p^{n}$. Now suppose $C$ has complex multication by $\mathcal{O}_{K}$ and $\mathcal{O}_{K}$ has two polarizations. It can easily be seen that $n \leq 2 h_{K}$. Thus in order to get a large extension degree we need a large class number, which is not possible. But we can use the algorithm for fields where $n>1$ is small.

A strong limitation of the CM-method is the fact that in general the invariants are not integers. As a consequence the class polynomial has rational coefficients. If the parameters chosen are not too big, the denominator can be found. This is the reason why our method works at all. But the denominators increase with the discriminant of the CM-field. It turns out that this makes our method infeasible if the class number gets too large. 
TABLE 5 .

\begin{tabular}{|c|c|c||c|c|}
\hline$D$ & $a$ & $b$ & degree & denominator \\
\hline 5 & 12 & 1 & 12 & $13^{8} 19^{8} 23^{8} 47^{8} 71^{8} 103^{8}$ \\
5 & 12 & 2 & 12 & $2^{2} 7^{8} 13^{8} 23^{8} 59^{8} 83^{8}$ \\
5 & 14 & 6 & 12 & $3^{1} 917^{12} 59^{12}$ \\
5 & 20 & 1 & 12 & $3^{5} 5^{6} 7^{8} 11^{8} 31^{8} 37^{8} 41^{8} 47^{8} 101^{8}$ \\
8 & 13 & 1 & 12 & $23^{8} 43^{8} 59^{8} 71^{8} 79^{8} 83^{8}$ \\
29 & 13 & 1 & 10 & $17^{12} 19^{12} 29^{12} 47^{12} 61^{12}$ \\
\hline
\end{tabular}

For demonstration we list a few CM-fields with class number 5 and 6 and the denominator of $H_{1}(X)$ in Table 5 .

\section{APPENDIX}

This algorithm is a generalization of (3.15) in [17.

Given a symmetric matrix $A \in \mathbb{R}^{k^{2}}$, a fixed vector $\epsilon \in\left(\mathbb{R}^{+}\right)^{k}$ and a constant $C \in \mathbb{R}$, it finds the set of vectors $x \in \mathbb{Z}^{k}$ such that

$$
(x+\epsilon)^{t} A(x+\epsilon) \leq C .
$$

First we compute an upper triangular matrix $\left(q_{i j}\right) \in \mathbb{R}^{k^{2}}$ with the property

$$
x^{t} A x=\sum_{i=1}^{k} q_{i i}\left(x_{i}+\sum_{j=i+1}^{k} q_{i j} x_{j}\right)^{2}
$$

for all $x \in \mathbb{R}^{k}$. For this algorithm, see $[17$.

For all $x \in \mathbb{Z}^{k}$ which satisfy the condition (4) we have, for $k \geq i \geq 1$,

$$
\begin{aligned}
& q_{i i}\left(\left(x_{i}+\epsilon_{i}\right)+\sum_{j=i+1}^{k} q_{i j}\left(x_{j}+\epsilon_{j}\right)\right)^{2} \\
& \quad \leq C-\sum_{\nu=i+1}^{k} q_{\nu \nu}\left(\left(x_{\nu}+\epsilon_{\nu}\right)+\sum_{j=\nu+1}^{k} q_{\nu j}\left(x_{j}+\epsilon_{j}\right)\right)^{2}=: T_{i} .
\end{aligned}
$$

Thus for $x_{k} \in \mathbb{Z}$ with

$$
\begin{aligned}
& \left|x_{k}\right| \leq\left(c / q_{k k}\right)^{\frac{1}{2}}-\epsilon_{k}, \text { if } x_{k} \text { is positive, } \\
& \left|x_{k}\right| \leq\left(c / q_{k k}\right)^{\frac{1}{2}}+\epsilon_{k}, \text { if } x_{k} \text { is negative, }
\end{aligned}
$$

we try to find all possiblities for $x_{k-1}$. 
For a fixed $x_{i+1}, \ldots, x_{k} \in \mathbb{Z}$, where

$$
\sum_{\nu=i+1}^{k} q_{\nu \nu}\left(x_{\nu}+\epsilon_{\nu}\right)+\sum_{j=\nu+1}^{k}\left(q_{\nu j}\left(x_{j}+\epsilon_{j}\right)\right)^{2} \leq T_{i+1},
$$

we obtain all possiblities for $x_{i}$ by

$$
\begin{array}{r}
-\left(T_{i} / q_{i i}\right)^{\frac{1}{2}}-U_{i}-\epsilon_{i} \leq x_{i} \leq\left(T_{i} / q_{i i}\right)^{\frac{1}{2}}-U_{i}-\epsilon_{i} \\
\text { where } U_{i}:=\sum_{j=i+1}^{k} q_{i j} x_{j} \text { for } k-1 \geq i \geq 1 .
\end{array}
$$

We get the following algorithm:

Finding all solutions $x \in \mathbb{Z}^{k}$ to $Q(\mathbf{x}+\epsilon) \leq C$

Input: Matrix $\left(q_{i j}\right)$, constant $C$, vector $\epsilon \in\left(\mathbb{R}^{+}\right)^{k}$.

Output: $\mathbf{x} \in \mathbb{Z}^{k}, \mathbf{x} \neq 0$, where $Q(\mathbf{x}+\epsilon) \leq C$

1: Let $T_{i}, U_{i}, x_{i}$ and $O S\left(x_{i}\right)$ be vectors of dimension $k$.

2: $i:=k ; T_{i}:=C ; U_{i}:=0$;

3: while $i \leq k$ do

bool_value $:=$ TRUE;

$Z:=\left(T_{i} / q_{i i}\right)^{\frac{1}{2}}$

$O S\left(x_{i}\right):=\left\lfloor Z-U_{i}-\epsilon_{i}\right\rfloor$;

$x_{i}:=\left\lceil-Z-U_{i}-\epsilon_{i}\right\rceil-1$;

while bool_value $=$ TRUE AND $i \leq \mathrm{k}$ do

$x_{i}:=x_{i}+1$;

if $\left(x_{i} \leq O S\left(x_{i}\right)\right)$ then

if $\mathrm{i}=1$ then

Output x;

else

$i:=i-1$;

$U_{i}:=\sum_{j=i+1}^{k} q_{i j}\left(x_{j}+\epsilon_{j}\right)$

$T_{i}:=T_{i-1}-q_{i+1, i+1}\left(x_{i+1}+\epsilon_{i+1}+U_{i+1}\right) ;$

bool_value $:=$ FALSE;

end if

else

$i:=i+1$;

end if

end while

end while

\section{ACKNOWLEDGMENTS}

I thank my supervisor Professor G. Frey for his support, help and encouragement. This work is part of my PHD-thesis.

Further I would like to thank K. Belabas for answering all my email-questions concerning the Pari-Library. Also I would like to thank all the people who helped me by pointing out interesting questions and reading the preliminary versions: $\mathrm{S}$. Galbraith, A. Menezes, K. Nguyen, H.-G. Rück, T. Schmidt, A. Stein. 
I am grateful to the NRW Verbundsprojekt Datensicherheit and the DFG (Graduiertenkolleg), who gave me the financial support making this work possible. I thank the Center for Applied Cryptographic Research in Waterloo, Ontario, for their hospitality.

\section{REFERENCES}

1. A.O.L. Atkin, The number of points on an elliptic curve modulo a prime, unpublished manuscript, 1991.

2. A.O.L. Atkin and F. Morain, Elliptic curves and primality proving, Math. Comp. 61 (1993), 29-68. MR 93m:11136

3. J. von zur Gathen and Victor Shoup, Computing Frobenius maps and factoring polynomials, Comput. Complexity 2 (1992), 187-224. MR 94d:12011

4. P. Gaudry and R. Harley, Counting points on hyperelliptic curves over finite fields, ANTS IV (2000), 313-332.

5. J.I.. Igusa, Arithmetic variety of moduli of genus two, Ann. of Math. 72 (1960), 612-649. MR 22:5637

6. D.E. Knuth, The art of computer programming vol.2, seminumerical algorithms, AddisonWeseley, 1981. MR 83i:68003

7. N. Koblitz, Primality of the number of points on an elliptic curve over a finite field, Pacific J. Math. 131 (1988), 157-165. MR 89h:11023

8. _ Hyperelliptic cryptosystems, J. Cryptology 1 (1989), 139-150. MR 90k:11165

9. S. Lang, Introduction to algebraic and abelian functions, 2nd ed., Springer-Verlag, 1982. MR 84m:14032

10. Complex multiplication, Springer-Verlag, 1983. MR 85f:11042

11. S. Louboutin and R. Okazaki, Determination of all non-normal quartic cm-fields and of all non-abelian normal octic cm-fields with class number one, Acta Arith. (1994), 47-62. MR 95g:11107

12. J.-F. Mestre, Construction des courbes de genre 2 a partir de leurs modules, Effective Methods in Algebraic Geometry (Castiglioncello, 1990), Prog. Math., Birkhäuser 94 (1991), 313-334. MR 92g: 14022

13. D. Mumford, Tata lecture on theta, vol. 1, Birkhäuser, 1983. MR 85h:14026

14. _ Tata lecture on theta, vol. 2, Birkhäuser, 1984. MR 86b:14017

15. R. Okazaki, On evaluation of L-functions over real quadratic fields, J. Math. Kyoto Univ. 31-4 (1991), 1125-1153. MR 93b:11154

16. S. Paulus and A. Stein, Comparing real and imaginary arithmetics for divisor class groups of hyperelliptic curves, ANTS III, LNCS 1423 (1998), 576-591. MR 2000i:11098

17. E. Pohst and H. Zassenhaus, Algorithmic algebraic number theory, Cambridge University Press, 1989. MR 92b:11074

18. S. Pohlig and M. Hellmann, An improved algorithm for computing logarithms over $G F(p)$ and its cryptographic significance, IEEE Trans. Inform. Theory IT-24 (1978), 106-110.

19. G. Shimura, Abelian varieties with complex multiplication and modular functions, revised ed., Princeton University Press, 1998. MR 99e:11076

20. J.A. Solinas, Generalized Mersenne numbers, Technical Reports, CACR, Waterloo (1999).

21. A.-M. Spallek, Kurven vom Geschlecht 2 und ihre Anwendung in Public-Key-Kryptosystemen, Ph.D. thesis, Institut für Experimentelle Mathematik, Universität GH Essen, 1994.

22. P. van Wamelen, Examples of genus two cm curves defined over the rationals, Math. Comp. 68 (1999), 307-320. MR 99c:11079

23. X. Wang, 2-dimensional simple factors of $J_{0}(N)$, Manuscripta Math. 87 (1995), 179-197. MR 96h:11059

24. H.J. Weber, Hyperelliptic simple factors of $J_{0}(N)$ with dimension at least 3, Experiment. Math. 6 (1997), 273-287. MR 99e:14054

Institute for Experimental Mathematics, University of Essen, D-45326 Essen, Germany

E-mail address: weng@exp-math.uni-essen.de 